\title{
Comparison of the two up-to-date sequencing technologies for genome assembly: HiFi reads of Pacbio Sequel II system and ultralong reads of Oxford Nanopore
}

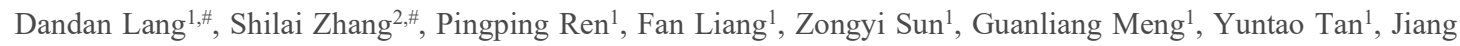
$\mathrm{Hu}^{1}$, Xiaokang Li, Qihua Lai, Lingling Han ${ }^{1}$, Depeng Wang ${ }^{1}$, Fengyi $\mathrm{Hu}^{2}$, Wen Wang ${ }^{3,4, *}$, Shanlin Liu $^{1,5^{*}}$

1. GrandOmics Biosciences, Beijing, 102200, China

2. State Key laboratory for Conservation and Utilization of Bio-Resources in Yunnan, Research Center for Perennial Rice Engineering and Technology of Yunnan, School of Agriculture, Yunnan University, Kunming, Yunnan, 650091, China

3. State Key Laboratory of Genetic Resources and Evolution, Kunming Institute of Zoology, Chinese Academy of Sciences, 650223 Kunming, Yunnan, China.

4. Center for Ecological and Environmental Sciences, Key Laboratory for Space Bioscience \& Biotechnology, Northwestern Polytechnical University, 710072 Xi'an, China.

5. Department of Entomology, College of Plant Protection, China Agricultural University, 100193 Beijing, China ${ }^{\#}$ Contribute equally

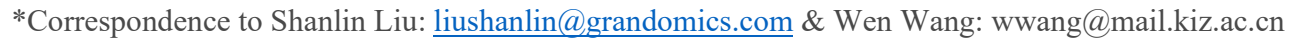

\section{Abstract}

The availability of reference genomes has revolutionized the study of biology. Multiple competing technologies have been developed to improve the quality and robustness of genome assemblies during the last decade. The two widely-used long read sequencing providers - Pacbio (PB) and Oxford Nanopore Technologies (ONT) - have recently updated their platforms: $\mathrm{PB}$ enable high throughput HiFi reads with base-level resolution with $>99 \%$ and ONT generated reads as long as $2 \mathrm{Mb}$. We applied the two up-to-date platforms to one single rice individual, and then compared the two assemblies to investigate the advantages and limitations of each. The results showed 
that ONT ultralong reads delivered higher contiguity producing a total of 18 contigs of which 10 were assembled into a single chromosome compared to that of 394 contigs and three chromosome-level contigs for the PB assembly. The ONT ultralong reads also prevented assembly errors caused by long repetitive regions for which we observed a total 44 genes of false redundancies and 10 genes of false losses in the PB assembly leading to over/under-estimations of the gene families in those long repetitive regions. We also noted that the PB HiFi reads generated assemblies with considerably less errors at the level of single nucleotide and small InDels than that of the ONT assembly which generated an average 1.06 errors per $\mathrm{Kb}$ assembly and finally engendered 1,475 incorrect gene annotations via altered or truncated protein predictions.

\section{Main text}

The availability of reference genomes has revolutionized the study of biology - many diseases found their causative alleles thanks to the high quality human reference genome $[1,2]$; the genomes of agricultural crops have tremendously accelerated our understanding on how artificial selection shaped plant traits and how, in turn, these plant traits may influence species interactions, e.g. phytophagous insects, in agriculture $[3,4]$. During the last decade, multiple competing technologies have been developed to improve the quality and robustness of genome assemblies [5-8], enabling genome reference collecting of the tree of life [9-11]. To date, a large number of genomes have been assembled by Third Generation Sequencing (TGS) technologies which can produce individual reads in the range of $10 \sim 100 \mathrm{kbs}$ or even longer [12-15]. Although the long-read still has a high error rate, it has been improving owing to the advances in sequencing chemistry and computational tools, e.g. Pacbio (PB) Single-molecule realtime (SMRT) sequencing platform released the Sequel II system of which the updated SMRT cell enabled high throughput HiFi reads using the circular consensus sequencing (CCS) mode to provide base-level resolution with $>99 \%$ single-molecule read accuracy [16]; while the Oxford Nanopore Technologies (ONT) launched its PromethION 
platform which can yield $>7 \mathrm{~Tb}$ per run and its ultralong sequencing application facilitates the achievement of complete genome - Telomere to Telomere (T2T) - by resolving long and complex repetitive regions for various species including Homo sapien [17]. Plenty of species begin to leverage the two cutting-edge sequencing systems; however, almost all chose one single sequencing system, either the PB or the ONT platform, to obtain their reference genomes $[15,18,19]$. Here we present one rice individual (variety 9311) that was sequenced and assembled independently using the two up-to-date systems, and then we compared the two assemblies to investigate the advantages and limitations of each.

Following DNA extraction from the rice sample, we sequenced the two extracts using ONT PromethION and PB Sequel II platforms, respectively. The PromethION generated a total of $92 \mathrm{~Gb}$ data with N50 of 41,473 bp and the Sequel II produced a total of $253 \mathrm{~Gb}$ data with each molecular fragment being sequenced 14.72 times on average and of an average length of 11,487 bp. We applied multiple software, including Canu1.9 [20], NextDenovo2.0-beta.1 (https://github.com/Nextomics/NextDenovo) and WTDBG2 [21] to assemble the rice genome for the ONT and PB dataset respectively. We selected the optimal assembly for each sequencing platform based on contig N50 and then got both polished (detailed in Supplementary Methods). The ONT assembly showed higher contiguity with a contig number of 18 and an N50 value of ca. $32 \mathrm{Mb}$ in comparison to a contig number of 394 and N50 of $17 \mathrm{Mb}$ for the PB assembly (Figure 1a \& Table S1). Ten and three out of the total 12 autosomes were assembled into a single contig in the ONT and PB assembly, respectively. We identified telomers and centromeres for both assemblies (Supplementary Methods) and found that seven of them reached a T2T level assembly with no gaps and no Ns in between (Table S2). A genome completeness assessment using BUSCOv3.1.0 [22] finds both assemblies performed well with the ONT having a tiny improvement $(98.62 \%$ vs $98.33 \%$, Table S3). We mapped both assemblies to a high-quality rice (R498) genome reference [23] using Minimap2 [24]. Both assemblies showed good collinearity (Figure S1) and the 
PB assembly contained more gaps in each chromosome compared to that of ONT (Figure 1a).

We then took Chr. 6 where ONT's one single contig $(32,367,127 \mathrm{bp})$ corresponded to nine contigs $(32,476,323 \mathrm{bp})$ of the $\mathrm{PB}$ assembly to investigate and visualize the incongruencies between them. For the nine contigs of PB assembled for the Chr. 6, four reached a length $\geq 6 \mathrm{Mb}$ and five had a length of merely $10-70 \mathrm{~kb}$. We investigated the three gaps where the top four PB contigs (named as PB-L1, PB-L2, PB-L3 and PB-L4 from 5' to 3'end, respectively) failed to connect (Figure 1b). We mapped the ONT ultralong reads to those gaps and confirmed their correctness through manual inspections by IGV plot [25](Figure S2). The gap \#1 between PB-L1 and PB-L2 reached a length of 74,888 bp. One of the short PB contigs (PB-S1, length of 70,208 bp) had an overlap of $\sim 10 \mathrm{~kb}$ with the 3'end of PB-L1, thus left the gap \#1 a region of 15,722 bp that PB failed to cover (Figure 1c). We further examined the sequences obtained by ONT in and flanking this gap. It showed that the overlapping and the gap regions represented two elements of $15 \mathrm{~kb}$ and $48 \mathrm{~kb}$ in length that, although have only one copy on Chr. 6, can find their duplications on Chr. 5 (Figure S3). Repetitive elements with such lengths go beyond the typical length generated by PB CCS, therefore the right path can hardly be disentangled from complicated string graphs. The gap \#2 between PB-L2 and PB-L3 characterized a region spanning up to $48 \mathrm{~kb}$ on the ONT assembly and is flanked by two tandem repeats of $14 \mathrm{~kb}$ in length. It was gone through by multiple ONT long reads (Figure S2), so can be successfully connected by the ONT assembly. The last gap between PB-L3 and PB-L4 can be connected by one short PB contig (PB-S2, 25,292 bp), which had 9,469 and 2,621 bp overlaps with 3'end of PB-L3 and 5'end of PB-L4, respectively. And it showed the same case as the gap \#2, containing three tandem duplicates of length $23 \mathrm{~kb}$ that failed to be connected by PB $\mathrm{HiFi}$ reads. We found a total of $107 \mathrm{~kb}$ redundancies and $15 \mathrm{~kb}$ gaps on Chr. 6 owing to PB's incorrect assemblies, which corresponded to an excess of 13 annotated genes (Figure 2, Table S4). The genome-wide misassembled regions accumulated to a length of $\sim 668 \mathrm{~kb}$ (534 kb redundancies and $134 \mathrm{~kb}$ gaps), hosting 54 annotated genes (44 
redundancies and 10 loss, Table S4). As PB assembly did not generate any single contigs that ONT broke into multiple segments, we cannot find a counter case for comparison.

In addition to those gaps that PB failed to connect, we noticed that there were a bunch of small-scale mismatches between the two assemblies. Firstly, we extracted the reciprocal matches $\geqslant 1 \mathrm{M}$ between the two assemblies for comparison using QUAST 5.0.2 [26]. Then, we mapped the PB HiFi reads to both genome assemblies to filter out the innate discrepancies derived from diploid heterozygous states under the assumption that HiFi reads provide high level single base accuracy (Supplementary Methods). It showed that the ONT assembly, although polished using 70x Illumina's shotgun reads, still contained a large number of small-scale mis-assemblies. In total, we found 210,993 single nucleotide errors and 211,517 InDels (Mean: 1.39 bp, Figure S4) accounting for an average number of 1.06 errors per $\mathrm{kb}$. However, instead of scattering evenly on the assembly, those errors formed into clusters (Figure S5). A further investigation for those regions showed $\sim 94 \%$ of them have a shotgun read coverage $\leq 5$, which explains why the last polishing step failed to fix those errors (Figure S6). About $7.48 \%$ of those errors located on exons and affected $\sim 2,415$ exons (1,475 genes) to translate correctly to amino acid sequences. We did note that, however, the errors of HiFi reads may be enriched in sequences with particular characteristics, rather than completely random, for example, regions like simple sequence repeats and long homopolymers (Supplementary Methods, Figure S7) which may exacerbate the above error statistics for the ONT assembly.

In conclusion, our study investigated genome assembly qualities between the two upto-date competing long read sequencing techniques - the PB's HiFi reads and the ONT's ultralong reads. It showed both techniques had their own merits with: (1) ONT ultralong reads delivered higher contiguity and prevented false redundancies caused by long repeats, which, in our case of rice genome, assembled 10 out of the 12 autosomes into one single contig, and (2) PB HiFi reads produced fewer errors at the level of single 
nucleotide and small InDels and obtained more than 1,400 genes that incorrectly annotated in the ONT assembly due to its error prone reads. Therefore, we suggest that further genomic studies, especially genome reference constructions, should leverage both techniques to lessen the impact of assembly errors and subsequent annotation mistakes rooted in each. There is also an urgent demand for improved assembly and error correction algorithms to fulfill this task.

\section{Availability of data and materials}

We have all the data including two genome assemblies and their corresponding raw reads deposited on NCBI under the project ID PRJNA600693.

\section{References}

1. Weischenfeldt J, Symmons O, Spitz F, Korbel JO: Phenotypic impact of genomic structural variation: insights from and for human disease. NAT REV GENET 2013, 14(2):125.

2. Fujimoto A, Furuta M, Totoki Y, Tsunoda T, Kato M, Shiraishi Y, Tanaka H, Taniguchi H, Kawakami $Y$, Ueno M: Whole-genome mutational landscape and characterization of noncoding and structural mutations in liver cancer. NAT GENET 2016, 48(5):500.

3. Saxena RK, Edwards D, Varshney RK: Structural variations in plant genomes. BRIEF FUNCT GENOMICS 2014, 13(4):296-307.

4. Chen YH, Gols R, Benrey B: Crop domestication and its impact on naturally selected trophic interactions. ANNU REV ENTOMOL 2015, 60:35-58.

5. Wheeler DA, Srinivasan M, Egholm M, Shen Y, Chen L, McGuire A, He W, Chen Y, Makhijani $\mathrm{V}$, Roth GT: The complete genome of an individual by massively parallel DNA sequencing. NATURE 2008, 452(7189):872.

6. Bentley DR, Balasubramanian S, Swerdlow HP, Smith GP, Milton J, Brown CG, Hall KP, Evers DJ, Barnes CL, Bignell HR: Accurate whole human genome sequencing using reversible terminator chemistry. NATURE 2008, 456(7218):53.

7. Pushkarev D, Neff NF, Quake SR: Single-molecule sequencing of an individual human genome. NAT BIOTECHNOL 2009, 27(9):847.

8. Rothberg JM, Hinz W, Rearick TM, Schultz J, Mileski W, Davey M, Leamon JH, Johnson K, Milgrew MJ, Edwards M: An integrated semiconductor device enabling non-optical genome sequencing. NATURE 2011, 475(7356):348.

9. Seberg O, Droege G, Barker K, Coddington JA, Funk V, Gostel M, Petersen G, Smith PP: Global Genome Biodiversity Network: saving a blueprint of the Tree of Life - a botanical perspective. ANN BOT-LONDON 2016, 118(3):393-399.

10. Mukherjee S, Seshadri R, Varghese NJ, Eloe-Fadrosh EA, Meier-Kolthoff JP, Göker M, Coates RC, Hadjithomas M, Pavlopoulos GA, Paez-Espino D: 1,003 reference genomes of bacterial and archaeal 
isolates expand coverage of the tree of life. NAT BIOTECHNOL 2017, 35(7):676.

11. Lewin HA, Robinson GE, Kress WJ, Baker WJ, Coddington J, Crandall KA, Durbin R, Edwards SV, Forest F, Gilbert MTP: Earth BioGenome Project: Sequencing life for the future of life. Proceedings of the National Academy of Sciences 2018, 115(17):4325-4333.

12. Chaisson MJ, Huddleston J, Dennis MY, Sudmant PH, Malig M, Hormozdiari F, Antonacci F, Surti $\mathrm{U}$, Sandstrom R, Boitano M: Resolving the complexity of the human genome using single-molecule sequencing. NATURE 2015, 517(7536):608.

13. VanBuren R, Bryant D, Edger PP, Tang H, Burgess D, Challabathula D, Spittle K, Hall R, Gu J, Lyons E: Single-molecule sequencing of the desiccation-tolerant grass Oropetium thomaeum. NATURE 2015, 527(7579):508.

14. Gordon D, Huddleston J, Chaisson MJ, Hill CM, Kronenberg ZN, Munson KM, Malig M, Raja A, Fiddes I, Hillier LW: Long-read sequence assembly of the gorilla genome. SCIENCE 2016, 352(6281):e344.

15. Jiao Y, Peluso P, Shi J, Liang T, Stitzer MC, Wang B, Campbell MS, Stein JC, Wei X, Chin C: Improved maize reference genome with single-molecule technologies. NATURE 2017, 546(7659):524.

16. Wenger AM, Peluso P, Rowell WJ, Chang P, Hall RJ, Concepcion GT, Ebler J, Fungtammasan A, Kolesnikov A, Olson ND: Accurate circular consensus long-read sequencing improves variant detection and assembly of a human genome. NAT BIOTECHNOL 2019, 37(10):1155-1162.

17. Miga KH, Koren S, Rhie A, Vollger MR, Gershman A, Bzikadze A, Brooks S, Howe E, Porubsky D, Logsdon GA: Telomere-to-telomere assembly of a complete human $\mathbf{X}$ chromosome. BioRxiv 2019:735928.

18. Loman NJ, Quick J, Simpson JT: A complete bacterial genome assembled de novo using only nanopore sequencing data. NAT METHODS 2015, 12(8):733.

19. Jain M, Koren S, Miga KH, Quick J, Rand AC, Sasani TA, Tyson JR, Beggs AD, Dilthey AT, Fiddes IT: Nanopore sequencing and assembly of a human genome with ultra-long reads. NAT BIOTECHNOL 2018, 36(4):338.

20. Koren S, Walenz BP, Berlin K, Miller JR, Bergman NH, Phillippy AM: Canu: scalable and accurate long-read assembly via adaptive $\mathbf{k}-$ mer weighting and repeat separation. GENOME RES 2017, 27(5):722-736.

21. Ruan J, Li H: Fast and accurate long-read assembly with wtdbg2. BioRxiv 2019:530972.

22. Seppey M, Manni M, Zdobnov EM: BUSCO: Assessing Genome Assembly and Annotation Completeness. In.: Springer; 2019: 227-245.

23. Du H, Yu Y, Ma Y, Gao Q, Cao Y, Chen Z, Ma B, Qi M, Li Y, Zhao X: Sequencing and de novo assembly of a near complete indica rice genome. NAT COMMUN 2017, 8:15324.

24. Li H: Minimap2: pairwise alignment for nucleotide sequences. BIOINFORMATICS 2018, 34(18):3094-3100.

25. Robinson JT, Thorvaldsdóttir H, Winckler W, Guttman M, Lander ES, Getz G, Mesirov JP: Integrative genomics viewer. NAT BIOTECHNOL 2011, 29(1):24.

26. Mikheenko A, Prjibelski A, Saveliev V, Antipov D, Gurevich A: Versatile genome assembly evaluation with QUAST-LG. BIOINFORMATICS 2018, 34(13):i142-i150. 
a
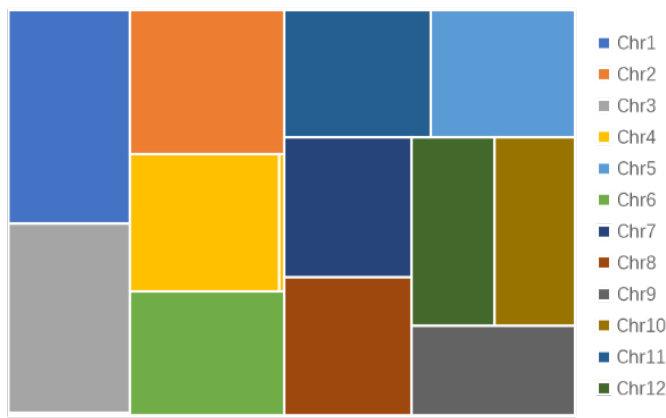

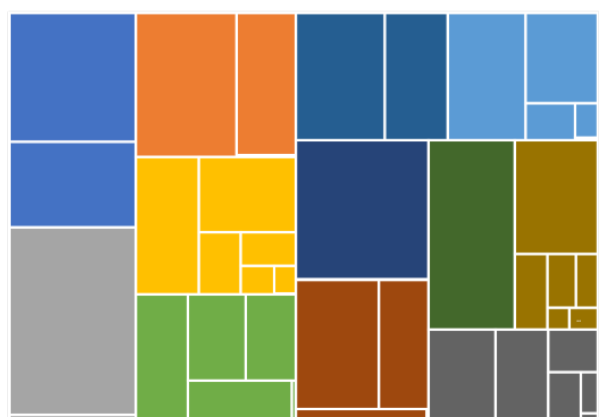
PB-L3 PB-S2 PB-S2 PB-L4

b

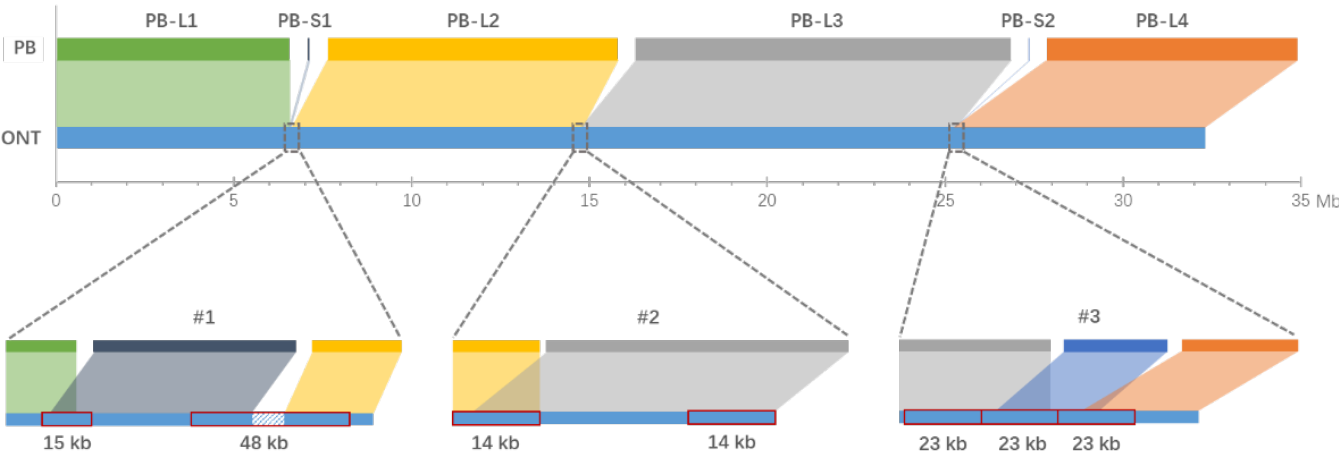

Figure 1. Contiguity of the ONT and PB assemblies. (a) Treemaps for contig length difference between the ONT (left) and PB (right) assembly; (b) The six PB contigs mapped to one ONT contig corresponding to Chr. 6; (c) Details of the three PB gaps. Red rectangles noted the repeat elements.

a
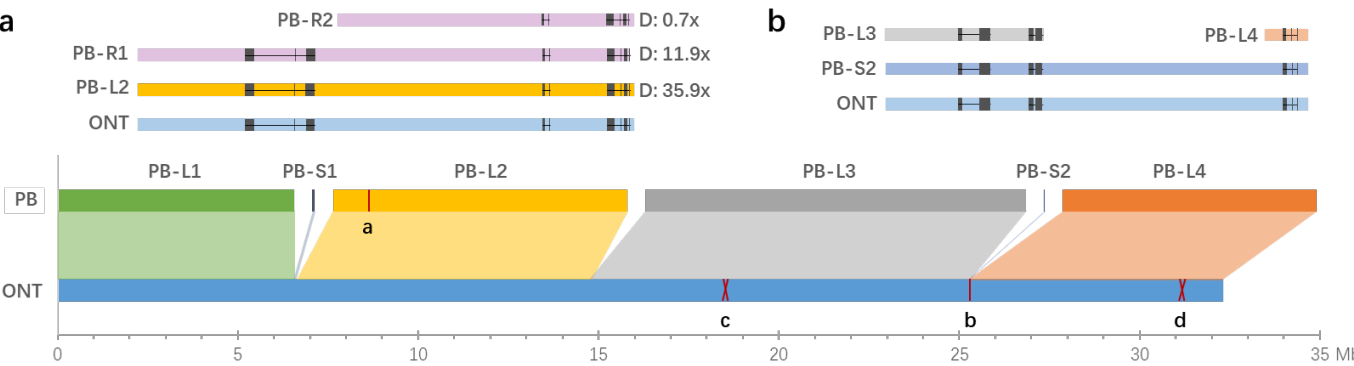

C PB-L3 GTCGTCGTCGCCGCACCTGAGCCGGATCCACCGCCCCCAAGCTCGTCGTCGCCGAGG

ValvalvalAlaAlaProGluProAspProProProproserserserserProArg ||||||||||||||||||||||||||||||||||

ValvalvalAlaAlaProGluProAspProProPro GlnAlaArgArgArgArg\#\#

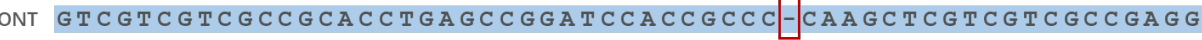

d PB-L4 CCCTGGGAGATAAGGAGAACGAGGTATTGAGGGTGCAAGGTATGTTATAAGGAT

ProTrpGluTyrIysGluAsnGluValIleGluGlyAlaArgTyrAlaTyrLysAsp

||||||||$!|!: !||||||||\quad !||||||||||||||||||||||||||||| \mid$

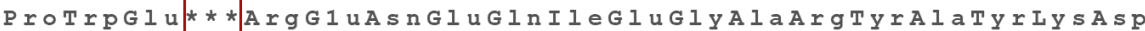

ONT C C T TGGAGISAAGGGAGAACGAGCAAATTGAGGGTGCAAGGTATGCTTATAAGGAT

Figure 2. Assembly errors in which genes can be annotated. (a) An example shows gene gains that caused by assembly redundancies, of which the PB-R1 and PB-R2 had a similarity level of $99.67 \%$ and 99.51\%, respectively, compared to the corresponding region on PB-L2, and " $\mathrm{D}$ " abbreviates from depth; (b) The gene redundancies caused by gaps that failed to be correctly connected by the PB assembly; (c) An example shows a 1-base deletion led to frameshift mistake for protein translation; (d) An example shows single base error led to stop codon gain and truncated protein translation. 